\title{
On the Inverse Problem of the Fractional Heat-Like Partial Differential Equations: Determination of the Source Function
}

\author{
Gülcan Özkum, ${ }^{1}$ Ali Demir, ${ }^{1}$ Sertaç Erman, ${ }^{1}$ Esra Korkmaz, ${ }^{2}$ and Berrak Özgür1 \\ ${ }^{1}$ Department of Mathematics, Science and Letter Faculty, Kocaeli University, Umuttepe Campus, 41380 Kocaeli, Turkey \\ ${ }^{2}$ Ardahan University, 75000 Ardahan, Turkey \\ Correspondence should be addressed to Gülcan Özkum; gulcan@kocaeli.edu.tr
}

Received 22 May 2013; Revised 12 September 2013; Accepted 12 September 2013

Academic Editor: H. Srivastava

Copyright (C) 2013 Gülcan Özkum et al. This is an open access article distributed under the Creative Commons Attribution License, which permits unrestricted use, distribution, and reproduction in any medium, provided the original work is properly cited.

The study in this paper mainly concerns the inverse problem of determining an unknown source function in the linear fractional differential equation with variable coefficient using Adomian decomposition method (ADM). We apply ADM to determine the continuous right hand side functions $f(x)$ and $f(t)$ in the heat-like diffusion equations $D_{t}^{\alpha} u(x, t)=h(x) u_{x x}(x, t)+f(x)$ and $D_{t}^{\alpha} u(x, t)=h(x) u_{x x}(x, t)+f(t)$, respectively. The results reveal that ADM is very effective and simple for the inverse problem of determining the source function.

\section{Introduction}

Fractional differential equations (FDEs) are obtained by generalizing differential equations to an arbitrary order. They are used to model physical systems with memory. Since FDEs have memory, nonlocal relations in space and time complex phenomena can be modeled by using these equations. Due to this fact, materials with memory and hereditary effects, fluid flow, rheology, diffusive transport, electrical networks, electromagnetic theory and probability, signal processing, and many other physical processes are diverse applications of FDEs. Since FDEs are used to model complex phenomena, they play a crucial role in engineering, physics, and applied mathematics. Therefore, they are generating an increasing interest from engineers and scientist in the recent years. As a result, FDEs are quite frequently encountered in different research areas and engineering applications [1].

The book written by Oldham and Spanier [2] played an outstanding role in the development of the fractional calculus. Also, it was the first book that was entirely devoted to a systematic presentation of the ideas, methods, and applications of the fractional calculus. Afterwards, several fundamental works on various aspects of the fractional calculus include extensive survey on fractional differential equations by Miller and Ross [3], Podlubny [4], and others. Further, several references to the books by Oldham and Spanier [2], Miller and Ross [3], and Podlubny [4] show that applied scientists need first of all an easy introduction to the theory of fractional derivatives and fractional differential equations, which could help them in their initial steps in adopting the fractional calculus as a method of research [5].

In general, FDEs do not have exact analytical solutions; hence, the approximate and numerical solutions of these equations are studied [6-8]. Analytical approximations for linear and nonlinear FDEs are obtained by variational iteration method, Adomian decomposition method, homotopy perturbation method, Lagrange multiplier method, BPs operational matrices method, and so forth. An effective and easyto-use method for solving such equations is needed. Large classes of linear and nonlinear differential equations, both ordinary and partial, can be solved by the Adomian decomposition method [9-12].

Solving an equation with certain data in a specified region is called direct problem. On the other hand, determining an unknown input by using output is called an inverse problem. This unknown input could be some coefficients, or it could be a source function in equation. Based on this unknown input the inverse problem is called inverse problem of coefficient identification or inverse problem of source identification, respectively. Generally inverse problems are 
ill-posed problems; that is, they are very sensitive to errors in measured input. In order to deal with this ill-posedness, regularization methods have been developed. Inverse problems have many practical applications such as geophysics, optics, quantum mechanics, astronomy, medical imaging and materials testing, X-ray tomography, and photoelasticity. Theoretical and applied aspects of inverse problems have been under intense study lately, especially for the fractional equation [13-16].

In this paper, we investigate inverse problems of the linear heat-like differential equations of fractional orders $D_{t}^{\alpha} u(x, t)=h(x) u_{x x}(x, t)+f(x)$ and $D_{t}^{\alpha} u(x, t)=$ $h(x) u_{x x}(x, t)+f(t)$ where the function $u(x, t)$ is assumed to be a causal function of time and space. Time fractional derivative operator $D_{t}^{\alpha}$ is considered as in Caputo sense [17]. We use the Adomian decomposition method $[9,10]$ to obtain source functions $f(x)$ and $f(t)$ under the initial and mixed boundary conditions. By this method, we determine the source functions $f(x)$ and $f(t)$ in a rapidly converging series form when they exist. Compared with previous researches $[10,12,18]$, the method we use in this paper is more effective and accurate.

The structure of this paper is given as follows. First, we give some basic definitions of fractional calculus. Inverse problem of finding the source function in one-dimensional fractional heat-like equations with mixed boundary conditions is given in Section 2. After that, we give some illustrative examples of this method for all cases in Section 3. Finally, the conclusion is given in Section 4.

1.1. Fractional Calculus. In this section, we give basic definitions and properties of the fractional calculus [17, 18].

Definition 1. A real function $f(x), x>0$, is said to be in the space $C_{\mu}, \mu \in \mathbb{R}$ if there exists a real number $p>\mu$ such that $f(x)=x^{p} f_{1}(x)$, where $f_{1}(x) \in C[0, \infty)$, and it is said to be in the space $C_{\mu}^{m}$ if $f^{(m)} \in C_{\mu}, m \in \mathbb{N}$.

Definition 2. The Riemann-Liouville fractional integral operator of order $\alpha \geq 0$, of a function $f \in C_{\mu}, \mu \geq-1$ is defined as

$$
\begin{gathered}
J^{\alpha} f(x)=\frac{1}{\Gamma(\alpha)} \int_{0}^{x}(x-t)^{\alpha-1} f(t) d t, \quad \alpha>0, x>0 \\
J^{0} f(x)=f(x) .
\end{gathered}
$$

Some of the basic properties of this operator are given as follows.

$$
\begin{aligned}
& \text { For } f \in C_{\mu}, \mu \geq-1, \alpha, \beta \geq 0 \text { and } \gamma>-1 \text { : } \\
& \begin{array}{r}
\text { (1) } J^{\alpha} J^{\beta} f(x)=J^{\alpha+\beta} f(x), \\
\text { (2) } J^{\alpha} J^{\beta} f(x)=J^{\beta} J^{\alpha} f(x), \\
\text { (3) } J^{\alpha} x^{\gamma}=\frac{\Gamma(\gamma+1)}{\Gamma(\alpha+\gamma+1)} x^{\alpha+\gamma} .
\end{array}
\end{aligned}
$$

The other properties can be found in [17].
Definition 3. The fractional derivative of $f(x)$ in the Caputo sense is defined as

$$
\begin{aligned}
D^{\alpha} f(x) & =J^{m-\alpha} D^{m} f(x) \\
& =\frac{1}{\Gamma(m-\alpha)} \int_{0}^{x}(x-t)^{m-\alpha-1} f^{(m)}(t) d t,
\end{aligned}
$$

where $m-1<\alpha \leq m, m \in \mathbb{N}, x>0, f \in C_{-1}^{m}$.

Useful properties of $D^{\alpha}$ are given as follows.

Lemma 4. If $m-1<\alpha \leq m, m \in \mathbb{N}$, and $f \in C_{\mu}^{m}, \mu \geq-1$, then

$$
\begin{gathered}
D^{\alpha} J^{\alpha} f(x)=f(x) \\
J^{\alpha} D^{\alpha} f(x)=f(x)-\sum_{k=0}^{m-1} f^{(k)}\left(0^{+}\right) \frac{x^{k}}{k !}, \quad x>0 .
\end{gathered}
$$

Since traditional initial and boundary conditions are allowed in problems including Caputo fractional derivatives, it is considered here. In this paper, we deal with the fractional heatlike equations where the unknown function $u=u(x, t)$ is an arbitrary function of time and space.

Definition 5. The Caputo time fractional derivative operator of order $\alpha>0$ is defined as follows where $m$ is the smallest integer that exceeds $\alpha$ :

$$
\begin{aligned}
& D_{t}^{\alpha} u(x, t) \\
& =\frac{\partial^{\alpha} u(x, t)}{\partial t^{\alpha}} \\
& = \begin{cases}\frac{1}{\Gamma(m-\alpha)} \\
\times \int_{0}^{t}(t-\tau)^{m-\alpha-1} \frac{\partial^{m} u(x, \tau)}{\partial t^{m}} d \tau, & \text { for } m-1<\alpha<m \\
\frac{\partial^{m} u(x, t)}{\partial t^{m}}, & \text { for } \alpha=m \in \mathrm{IN} .\end{cases}
\end{aligned}
$$

For more details about Caputo fractional differential operator, we refer to [17].

Definition 6. The Mittag-Leffler function with twoparameters is defined by the series expansion as shown below, where the real part of $\alpha$ is strictly positive [19]

$$
E_{\alpha, \beta}(z)=\sum_{n=0}^{\infty} \frac{z^{n}}{\Gamma(\alpha n+\beta)} .
$$

\section{Inverse Problem of Determining Source Function}

In this section, we deal with inverse problem of finding the source function, in one-dimensional fractional heat-like equations with mixed boundary conditions. To determine the unknown source function we have developed new methods through $\mathrm{ADM}$ as in the following subsections. 
2.1. Determination of Unknown Source Functions Depending on $x$. We consider the following inverse problem of determining the source function $f(x)$ :

$$
\begin{aligned}
D_{t}^{\alpha} u(x, t) & =h(x) u_{x x}(x, t)+f(x), \\
x>0, \quad t>0, \quad 0<\alpha \leq 1, & \\
u(x, 0) & =f_{1}(x), \\
u(0, t) & =h_{1}(t), \\
u_{x}(0, t) & =h_{2}(t),
\end{aligned}
$$

where the functions $h_{1}(t), h_{2}(t) \in C^{\infty}[0, \infty)$ and $h(x), f(x)$, $f_{1}(x) \in C^{\infty}[0, \infty)$. In order to determine the source function for this kind of inverse problems, we apply ADM. First, we apply the time-dependent Riemann-Liouville fractional integral operator $J_{t}^{\alpha}$ to both sides of (7) to get rid of fractional derivative $D_{t}^{\alpha}$ as shown below:

$$
J_{t}^{\alpha} D_{t}^{\alpha} u(x, t)=J_{t}^{\alpha}\left(h(x) u_{x x}(x, t)\right)+J_{t}^{\alpha} f(x) .
$$

Then we get

$$
u(x, t)=u(x, 0)+J_{t}^{\alpha} f(x)+J_{t}^{\alpha}\left(h(x) u_{x x}(x, t)\right)
$$

In $\mathrm{ADM}$ the solution $u(x, t)$ is written in the following series form [9]:

$$
u(x, t)=\sum_{n=0}^{\infty} u_{n}(x, t)
$$

where $u$ and $u_{n}, n \in \mathbb{N}$, are defined in $C^{\infty}[0, \infty) \times C_{\mu}^{1}[0, \infty)$. After substituting the decomposition (10) into (9) and setting the recurrence scheme as follows:

$$
\begin{gathered}
u_{0}(x, t)=u(x, 0)+J_{t}^{\alpha} f(x), \\
u_{n+1}(x, t)=J_{t}^{\alpha}\left(h(x)\left(u_{n}\right)_{x x}(x, t)\right), \quad n=0,1, \ldots,
\end{gathered}
$$

we get ADM polynomials below

$$
\begin{aligned}
& u_{0}(x, t)=f_{1}(x)+f(x) \frac{t^{\alpha}}{\Gamma(\alpha+1)} \\
& u_{1}(x, t)=J_{t}^{\alpha}\left(h(x)\left(u_{0}\right)_{x x}(x)\right)
\end{aligned}
$$

$$
\begin{gathered}
=h(x) f_{1}^{\prime \prime}(x) \frac{t^{\alpha}}{\Gamma(\alpha+1)} \\
+h(x) f^{\prime \prime}(x) \frac{t^{2 \alpha}}{\Gamma(2 \alpha+1)}, \\
\begin{array}{c}
u_{2}(x, t) \quad \\
=J_{t}^{\alpha}\left(h(x)\left(u_{1}\right)_{x x}(x)\right) \\
=h(x)\left\{\left[h^{\prime \prime}(x) f_{1}^{\prime}(x)+h^{\prime}(x) f_{1}^{\prime \prime}(x)\right.\right. \\
\left.+h^{\prime}(x) f_{1}^{\prime \prime \prime}(x)+h(x) f_{1}^{(i v)}(x)\right] \frac{t^{2 \alpha}}{\Gamma(2 \alpha+1)} \\
+\left[h^{\prime \prime}(x) f^{\prime \prime}(x)+2 h^{\prime}(x) f^{\prime \prime \prime}(x)\right. \\
\left.\left.+h(x) f^{(i v)}(x)\right] \frac{t^{3 \alpha}}{\Gamma(3 \alpha+1)}\right\}
\end{array}
\end{gathered}
$$

After writing these polynomials in (10), the solution $u(x, t)$ is given by

$$
\begin{aligned}
& u(x, t) \\
& =f_{1}(x)+f(x) \frac{t^{\alpha}}{\Gamma(\alpha+1)}+h(x) f_{1}^{\prime \prime}(x) \frac{t^{\alpha}}{\Gamma(\alpha+1)} \\
& +h(x) f^{\prime \prime}(x) \frac{t^{2 \alpha}}{\Gamma(2 \alpha+1)} \\
& +h(x)\left\{\left[h^{\prime \prime}(x) f_{1}^{\prime}(x)+h^{\prime}(x) f_{1}^{\prime \prime}(x)\right.\right. \\
& \left.\quad+h^{\prime}(x) f_{1}^{\prime \prime \prime}(x)+h(x) f_{1}^{(i v)}(x)\right] \frac{t^{2 \alpha}}{\Gamma(2 \alpha+1)} \\
& +\left[h^{\prime \prime}(x) f^{\prime \prime}(x)+2 h^{\prime}(x) f^{\prime \prime \prime}(x)\right. \\
& \left.\left.+h(x) f^{(i v)}(x)\right] \frac{t^{3 \alpha}}{\Gamma(3 \alpha+1)}\right\}+\cdots
\end{aligned}
$$

If we arrange it with respect to like powers of $t$, then we get

$$
\begin{aligned}
& u(x, t) \\
& =f_{1}(x)+\left[f(x)+h(x) f_{1}^{\prime \prime}(x)\right] \frac{t^{\alpha}}{\Gamma(\alpha+1)} \\
& +h(x)\left\{\left[f^{\prime \prime}(x)+h^{\prime \prime}(x) f_{1}^{\prime}(x)+h^{\prime}(x) f_{1}^{\prime \prime}(x)\right.\right. \\
& \left.+h^{\prime}(x) f_{1}^{\prime \prime \prime}(x)+h(x) f_{1}^{(i v)}(x)\right] \frac{t^{2 \alpha}}{\Gamma(2 \alpha+1)}
\end{aligned}
$$




$$
\begin{aligned}
& +\left[h^{\prime \prime}(x) f^{\prime \prime}(x)+2 h^{\prime}(x) f^{\prime \prime \prime}(x)\right. \\
& \left.\left.\quad+h(x) f^{(i v)}(x)\right] \frac{t^{3 \alpha}}{\Gamma(3 \alpha+1)}\right\}+\cdots .
\end{aligned}
$$

To determine the unknown source function, first we expand the boundary conditions $u(0, t)=h_{1}(t)$ and $u_{x}(0, t)=$ $h_{2}(t)$ into the following series for the space whose bases are $\left\{t^{n \alpha} / \Gamma(n \alpha+1)\right\}_{n=0}^{\infty}, 0<\alpha \leq 1$ :

$$
\begin{aligned}
& h_{1}(t)=h_{1}(0)+h_{1}^{\prime}(0) \frac{t^{\alpha}}{\Gamma(\alpha+1)}+h_{1}^{\prime \prime}(0) \frac{t^{2 \alpha}}{\Gamma(2 \alpha+1)}+\cdots, \\
& h_{2}(t)=h_{2}(0)+h_{2}^{\prime}(0) \frac{t^{\alpha}}{\Gamma(\alpha+1)}+h_{2}^{\prime \prime}(0) \frac{t^{2 \alpha}}{\Gamma(2 \alpha+1)}+\cdots .
\end{aligned}
$$

On the other hand, if we rewrite the boundary conditions $u(0, t)$ and $u_{x}(0, t)$ from (14), then we have

$$
\begin{aligned}
h_{1}(t)=f_{1}(0)+\left[f(0)+h(0) f_{1}^{\prime \prime}(0)\right] \frac{t^{\alpha}}{\Gamma(\alpha+1)} \\
+h(0)\left\{\left[f^{\prime \prime}(0)+h^{\prime \prime}(0) f_{1}^{\prime}(0)+h^{\prime}(0) f_{1}^{\prime \prime}(0)\right.\right. \\
\left.+h^{\prime}(0) f_{1}^{\prime \prime \prime}(0)+h(0) f_{1}^{(i v)}(0)\right] \frac{t^{2 \alpha}}{\Gamma(2 \alpha+1)} \\
+\left[h^{\prime \prime}(0) f^{\prime \prime}(0)+2 h^{\prime}(0) f^{\prime \prime \prime}(0)\right. \\
\left.\left.+h(0) f^{(i v)}(0)\right] \frac{t^{3 \alpha}}{\Gamma(3 \alpha+1)}\right\}+\cdots,
\end{aligned}
$$

$$
\begin{aligned}
h_{2}(t)=f_{1}^{\prime}(0)+\left[f^{\prime}(0)+h^{\prime}(0) f_{1}^{\prime \prime}(0)\right. & \\
& \left.+h(0) f_{1}^{\prime \prime \prime}(0)\right] \frac{t^{\alpha}}{\Gamma(\alpha+1)} \\
+\left\{h ^ { \prime } ( 0 ) \left[f^{\prime \prime}(0)+h^{\prime \prime}(0) f_{1}^{\prime}(0)+h^{\prime}(0) f_{1}^{\prime \prime}(0)\right.\right. & \\
& \left.+h^{\prime}(0) f_{1}^{\prime \prime \prime}(0)+h(0) f_{1}^{(i v)}(0)\right] \\
+h(0)[ & f^{\prime \prime \prime}(0)+h^{\prime \prime \prime}(0) f_{1}^{\prime}(0) \\
& +2 h^{\prime \prime}(0) f_{1}^{\prime \prime}(0) \\
& +h^{\prime}(0) f_{1}^{\prime \prime \prime}(0)+h^{\prime \prime}(0) f_{1}^{\prime \prime \prime}(0) \\
& +2 h^{\prime}(0) f_{1}^{(i v)}(0) \\
& \left.\left.+h(0) f_{1}^{(v)}(0)\right]\right\} \frac{t^{2 \alpha}}{\Gamma(2 \alpha+1)}
\end{aligned}
$$

$$
\begin{aligned}
&+\left\{h ^ { \prime } ( 0 ) \left[h^{\prime \prime}(0) f^{\prime \prime}(0)+2 h^{\prime}(0) f^{\prime \prime \prime}(0)\right.\right. \\
&+\left.h(0) f^{(i v)}(0)\right] \\
&+h(0)\left[h^{\prime \prime \prime}(0) f^{\prime \prime}(0)+3 h^{\prime \prime}(0) f^{\prime \prime \prime}(0)\right. \\
&+3 h^{\prime}(0) f^{(i v)}(0) \\
&\left.\left.+h(0) f^{(v)}(0)\right]\right\} \frac{t^{3 \alpha}}{\Gamma(3 \alpha+1)}+\cdots .
\end{aligned}
$$

Equating (15) and (17) yields the following:

$$
\begin{gathered}
h_{1}(0)=f_{1}(0) \\
h_{1}^{\prime}(0)=f(0)+h(0) f_{1}^{\prime \prime}(0), \\
h_{1}^{\prime \prime}(0)=h(0)\left[f^{\prime \prime}(0)+h^{\prime \prime}(0) f_{1}^{\prime}(0)+h^{\prime}(0) f_{1}^{\prime \prime}(0)\right. \\
\left.+h^{\prime}(0) f_{1}^{\prime \prime \prime}(0)+h(0) f_{1}^{(i v)}(0)\right],
\end{gathered}
$$

and equating (16) and (18) yields the following:

$$
\begin{gathered}
h_{2}(0)=f_{1}^{\prime}(0) \\
h_{2}^{\prime}(0)=f^{\prime}(0)+h^{\prime}(0) f_{1}^{\prime \prime}(0)+h(0) f_{1}^{\prime \prime \prime}(0) \\
h_{2}^{\prime \prime}(0)=h^{\prime}(0)\left[f^{\prime \prime}(0)+h^{\prime \prime}(0) f_{1}^{\prime}(0)+h^{\prime}(0) f_{1}^{\prime \prime}(0)\right. \\
\left.+h^{\prime}(0) f_{1}^{\prime \prime \prime}(0)+h(0) f_{1}^{(i v)}(0)\right] \\
+h(0)\left[f^{\prime \prime \prime}(0)+h^{\prime \prime \prime}(0) f_{1}^{\prime}(0)+2 h^{\prime \prime}(0) f_{1}^{\prime \prime}(0)\right. \\
+h^{\prime}(0) f_{1}^{\prime \prime \prime}(0)+h^{\prime \prime}(0) f_{1}^{\prime \prime \prime}(0) \\
\left.+2 h^{\prime}(0) f_{1}^{(i v)}(0)+h(0) f_{1}^{(v)}(0)\right]
\end{gathered}
$$

Using the above data in the following Taylor series expansion of unknown function $f(x)$ we get

$$
f(x)=f(0)+f^{\prime}(0) x+f^{\prime \prime}(0) \frac{x^{2}}{2 !}+f^{\prime \prime \prime}(0) \frac{x^{3}}{3 !}+\cdots
$$

Consequently, we determine $f(x)$ as follows:

$$
\begin{aligned}
f(x)= & {\left[h_{1}^{\prime}(0)-h(0) f_{1}^{\prime \prime}(0)\right] } \\
& +\left[h_{2}^{\prime}(0)-h^{\prime}(0) f_{1}^{\prime \prime}(0)-h(0) f_{1}^{\prime \prime \prime}(0)\right] x
\end{aligned}
$$




$$
\begin{aligned}
& +\left[\frac{h_{1}^{\prime \prime}(0)}{h(0)}-h^{\prime \prime}(0) f_{1}^{\prime}(0)-h^{\prime}(0) f_{1}^{\prime \prime}(0)\right. \\
& \left.\quad-h^{\prime}(0) f_{1}^{\prime \prime \prime}(0)-h(0) f_{1}^{(i v)}(0)\right] \frac{x^{2}}{2 !}+\cdots,
\end{aligned}
$$

where $h(0) \neq 0$.

2.2. Determination of Unknown Source Functions Depending on $t$. We consider the following inverse problem of determining the source function $f(t)$ :

$$
\begin{aligned}
D_{t}^{\alpha} u(x, t) & =h(x) u_{x x}(x, t)+f(t), \\
x>0, \quad t>0, \quad 0<\alpha \leq 1, & \\
u(x, 0) & =f_{1}(x), \\
u(0, t) & =h_{1}(t), \\
u_{x}(0, t) & =h_{2}(t),
\end{aligned}
$$

where $h_{1}(t), h_{2}(t) \in C^{\infty}[0, \infty), h(x), f_{1}(x) \in C^{\infty}[0, \infty)$, and $f(t) \in C_{\mu}^{1}[0, \infty), \mu \geq-1$. As in the previous case, we apply ADM to determine the unknown function $f(t)$.

First, to reduce the problem, we define new functions in the following form:

$$
\begin{gathered}
w(t)=J_{t}^{\alpha} f(t) \\
u(x, t)=v(x, t)+w(t) .
\end{gathered}
$$

Then the reduced problem is given as follows:

$$
D_{t}^{\alpha} v(x, t)=h(x) v_{x x}(x, t)
$$

with the following initial and mixed boundary conditions

$$
\begin{gathered}
v(x, 0)=f_{1}(x)-w(0), \\
v(0, t)=h_{1}(t)-w(t), \\
v_{x}(0, t)=h_{2}(t) .
\end{gathered}
$$

By using ADM as in the previous section, we determine the function $w(t)$ which leads to the source function $f(t)$. Let us apply $J_{t}^{\alpha}$ to both sides of (26) as shown below

$$
J_{t}^{\alpha} D_{t}^{\alpha} v(x, t)=J_{t}^{\alpha}\left(h(x) v_{x x}(x, t)\right) .
$$

Then we get

$$
v(x, t)=v(x, 0)+J_{t}^{\alpha}\left(h(x) v_{x x}(x, t)\right) .
$$

Now we define the solution $v(x, t)$ by the following decomposition series according to ADM

$$
v(x, t)=\sum_{n=0}^{\infty} v_{n}(x, t) .
$$

Substituting (30) into (29), we obtain

$$
\begin{gathered}
v_{0}(x, t)=v(x, 0), \\
v_{n+1}(x, t)=J_{t}^{\alpha}\left(h(x)\left(v_{n}\right)_{x x}(x, t)\right), \quad n=0,1, \ldots .
\end{gathered}
$$

Hence, the recurrence scheme is obtained as follows:

$$
v_{0}(x, t)=v(x, 0)=f_{1}(x)-w(0),
$$

$$
\begin{gathered}
v_{1}(x, t)=J_{t}^{\alpha}\left(h(x)\left(v_{0}\right)_{x x}(x)\right)=h(x) f_{1}^{\prime \prime}(x) \frac{t^{\alpha}}{\Gamma(\alpha+1)}, \\
v_{2}(x, t)=J_{t}^{\alpha}\left(h(x)\left(v_{1}\right)_{x x}(x)\right)=h^{2}(x) f_{1}^{(i v)}(x) \frac{t^{2 \alpha}}{\Gamma(2 \alpha+1)},
\end{gathered}
$$

Consequently, from (30), the solution $v(x, t)$ is given as shown below

$$
\begin{aligned}
v(x, t)= & v_{0}(x, t)+v_{1}(x, t)+v_{2}(x, t)+\cdots \\
= & f_{1}(x)-w(0)+h(x) f_{1}^{\prime \prime}(x) \frac{t^{\alpha}}{\Gamma(\alpha+1)} \\
& +h^{2}(x) f_{1}^{(i v)}(x) \frac{t^{2 \alpha}}{\Gamma(2 \alpha+1)}+\cdots .
\end{aligned}
$$

By using the boundary condition $v(0, t)=h_{1}(t)+w(t)$ and $w(0)=0$, we have

$$
\begin{aligned}
w(t)= & f_{1}(0)-h_{1}(t)+h(0) f_{1}^{\prime \prime}(0) \frac{t^{\alpha}}{\Gamma(\alpha+1)} \\
& +h^{2}(0) f_{1}^{(i v)}(0) \frac{t^{2 \alpha}}{\Gamma(2 \alpha+1)}+\cdots
\end{aligned}
$$

which implies the following:

$$
\begin{aligned}
J_{t}^{\alpha} f(t)= & f_{1}(0)-h_{1}(t)+h(0) f_{1}^{\prime \prime}(0) \frac{t^{\alpha}}{\Gamma(\alpha+1)} \\
& +h^{2}(0) f_{1}^{(i v)}(0) \frac{t^{2 \alpha}}{\Gamma(2 \alpha+1)}+\cdots
\end{aligned}
$$

Since $D_{t}^{\alpha} w(x, t)=D_{t}^{\alpha} J_{t}^{\alpha} f(t)=f(t)$, we obtain the source function $f(t)$ as follows:

$$
\begin{array}{r}
f(t)=D_{t}^{\alpha}\left[f_{1}(x)-h_{1}(t)+h(x) f_{1}^{\prime \prime}(x) \frac{t^{\alpha}}{\Gamma(\alpha+1)}\right. \\
\left.+h^{2}(x) f_{1}^{(i v)}(x) \frac{t^{2 \alpha}}{\Gamma(2 \alpha+1)}+\cdots\right] .
\end{array}
$$

\section{Examples}

Example 1. We consider the inverse problem of determining source function $f(x)$ in the following one-dimensional fractional heat-like PDE:

$$
D_{t}^{\alpha} u(x, t)=2 u_{x x}(x, t)+f(x), \quad x>0,0<\alpha \leq 1, t>0,
$$


subject to the following initial and nonhomogeneous mixed boundary conditions:

$$
\begin{gathered}
u(x, 0)=e^{x}+\sin x, \\
u(0, t)=e^{2 t}, \\
u_{x}(0, t)=e^{2 t+1} .
\end{gathered}
$$

Now, let us apply the time-dependent Riemann Liouville fractional integral operator $J_{t}^{\alpha}$ to both sides of (37)

$$
J_{t}^{\alpha} D_{t}^{\alpha} u(x, t)=2 J_{t}^{\alpha} u_{x x}(x, t)+J_{t}^{\alpha} f(x)
$$

which implies

$$
u(x, t)-u(x, 0)=2 J_{t}^{\alpha} u_{x x}(x, t)+f(x) J_{t}^{\alpha}(1) .
$$

Then, from the initial condition we get

$$
u(x, t)=e^{x}+\sin x+f(x) \frac{t^{\alpha}}{\Gamma(\alpha+1)}+2 J_{t}^{\alpha} u_{x x}(x, t) .
$$

Now, we apply ADM to the problem. In (41), the sum of the first three terms is identified as $u_{0}$. So

$$
\begin{aligned}
& u_{0}=e^{x}+\sin x+f(x) \frac{t^{\alpha}}{\Gamma(\alpha+1)}, \\
& u_{k+1}=2 J_{t}^{\alpha}\left(u_{k}\right)_{x x}(x, t), \quad k \geq 0 .
\end{aligned}
$$

For $k=0$, we have

$$
\begin{aligned}
u_{1}= & 2 J_{t}^{\alpha}\left(u_{0}\right)_{x x}(x, t) \\
= & 2 e^{x} \frac{t^{\alpha}}{\Gamma(\alpha+1)}-2 \sin x \frac{t^{\alpha}}{\Gamma(\alpha+1)} \\
& +2 f^{\prime \prime}(x) \frac{t^{2 \alpha}}{\Gamma(2 \alpha+1)}+\cdots
\end{aligned}
$$

similarly, for $k=1$, we have

$$
\begin{aligned}
u_{2}= & 2 J_{t}^{\alpha}\left(u_{1}\right)_{x x}(x, t) \\
= & 4 e^{x} \frac{t^{2 \alpha}}{\Gamma(2 \alpha+1)}+4 \sin x \frac{t^{2 \alpha}}{\Gamma(2 \alpha+1)} \\
& +4 f^{(i v)}(x) \frac{t^{3 \alpha}}{\Gamma(3 \alpha+1)}+\cdots
\end{aligned}
$$

and for $k=2$, we have

$$
\begin{aligned}
u_{3}= & 2 J_{t}^{\alpha}\left(u_{2}\right)_{x x}(x, t) \\
= & 8 e^{x} \frac{t^{3 \alpha}}{\Gamma(3 \alpha+1)}-8 \sin x \frac{t^{3 \alpha}}{\Gamma(3 \alpha+1)} \\
& +8 f^{(v i)}(x) \frac{t^{4 \alpha}}{\Gamma(4 \alpha+1)}+\cdots
\end{aligned}
$$

Then using ADM polynomials, we get the solution $u(x, t)$ as follows:

$$
\begin{aligned}
u(x, t)= & u_{0}+u_{1}+u_{2}+u_{3}+\cdots \\
= & e^{x}+\sin x+f(x) \frac{t^{\alpha}}{\Gamma(\alpha+1)}+2 e^{x} \frac{t^{\alpha}}{\Gamma(\alpha+1)} \\
& -2 \sin x \frac{t^{\alpha}}{\Gamma(\alpha+1)}+2 f^{\prime \prime}(x) \frac{t^{2 \alpha}}{\Gamma(2 \alpha+1)} \\
& +4 e^{x} \frac{t^{2 \alpha}}{\Gamma(2 \alpha+1)}+4 \sin x \frac{t^{2 \alpha}}{\Gamma(2 \alpha+1)} \\
& +4 f^{(i v)}(x) \frac{t^{3 \alpha}}{\Gamma(3 \alpha+1)}+8 e^{x} \frac{t^{3 \alpha}}{\Gamma(3 \alpha+1)} \\
& -8 \sin x \frac{t^{3 \alpha}}{\Gamma(3 \alpha+1)}+8 f^{(v i)}(x) \frac{t^{4 \alpha}}{\Gamma(4 \alpha+1)}+\cdots
\end{aligned}
$$

After arranging it according to like powers of $t$, we have

$$
\begin{aligned}
u(x, t)= & e^{x}+\sin x+\frac{t^{\alpha}}{\Gamma(\alpha+1)}\left[f(x)+2 e^{x}-2 \sin x\right] \\
& +\frac{t^{2 \alpha}}{\Gamma(2 \alpha+1)}\left[2 f^{\prime \prime}(x)+4 e^{x}+4 \sin x\right] \\
& +\frac{t^{3 \alpha}}{\Gamma(3 \alpha+1)}\left[4 f^{(i v)}(x)+8 e^{x}-8 \sin x\right] \\
& +\frac{t^{4 \alpha}}{\Gamma(4 \alpha+1)}\left[8 f^{(v i)}(x)+16 e^{x}+16 \sin x\right]+\cdots
\end{aligned}
$$

Now, by applying the boundary condition given in (38), we obtain

$$
\begin{aligned}
u(0, t)= & 1+\frac{t^{\alpha}}{\Gamma(\alpha+1)}[f(0)+2]+\frac{t^{2 \alpha}}{\Gamma(2 \alpha+1)}\left[2 f^{\prime \prime}(0)+4\right] \\
& +\frac{t^{3 \alpha}}{\Gamma(3 \alpha+1)}\left[4 f^{(i v)}(0)+8\right] \\
& +\frac{t^{4 \alpha}}{\Gamma(4 \alpha+1)}\left[8 f^{(v i)}(0)+16\right]+\cdots
\end{aligned}
$$

From (15), it must be equal to the following Taylor series expansion of $e^{2 t}$ in the space whose bases are $\left\{t^{n \alpha} / \Gamma(n \alpha+1)\right\}_{n=0}^{\infty}, 0<\alpha \leq 1$ :

$$
\begin{aligned}
e^{2 t}= & 1+2 \frac{t^{\alpha}}{\Gamma(\alpha+1)}+4 \frac{t^{2 \alpha}}{\Gamma(2 \alpha+1)} \\
& +8 \frac{t^{3 \alpha}}{\Gamma(3 \alpha+1)}+16 \frac{t^{4 \alpha}}{\Gamma(4 \alpha+1)}+\cdots
\end{aligned}
$$

Hence, from the equality of the coefficients of corresponding terms, we get

$$
f(0)=f^{\prime \prime}(0)=f^{(i v)}(0)=f^{(v i)}(0)=\cdots=0 .
$$


From (47), we have

$$
\begin{aligned}
u_{x}(x, t)= & e^{x}+\cos x+\frac{t^{\alpha}}{\Gamma(\alpha+1)}\left[f^{\prime}(x)+2 e^{x}-2 \cos x\right] \\
& +\frac{t^{2 \alpha}}{\Gamma(2 \alpha+1)}\left[2 f^{\prime \prime \prime}(x)+4 e^{x}+4 \cos x\right] \\
& +\frac{t^{3 \alpha}}{\Gamma(3 \alpha+1)}\left[4 f^{(v)}(x)+8 e^{x}-8 \cos x\right] \\
& +\frac{t^{4 \alpha}}{\Gamma(4 \alpha+1)}\left[8 f^{(v i i)}(x)+16 e^{x}+16 \cos x\right]+\cdots
\end{aligned}
$$

So,

$$
\begin{aligned}
u_{x}(0, t)= & 2+\frac{t^{\alpha}}{\Gamma(\alpha+1)} f^{\prime}(0)+\frac{t^{2 \alpha}}{\Gamma(2 \alpha+1)}\left[2 f^{\prime \prime \prime}(0)+8\right] \\
& +\frac{t^{3 \alpha}}{\Gamma(3 \alpha+1)}\left[4 f^{(v)}(0)\right] \\
& +\frac{t^{4 \alpha}}{\Gamma(4 \alpha+1)}\left[8 f^{(v i i)}(0)+32\right]+\cdots .
\end{aligned}
$$

From the derivative boundary condition given in (38), it must be equal to the following series expansion of $e^{2 t}+1$ in the space whose bases are $\left\{t^{n \alpha} / \Gamma(n \alpha+1)\right\}_{n=0}^{\infty}, 0<\alpha \leq 1$ :

$$
\begin{aligned}
e^{2 t}+1= & 2+2 \frac{t^{\alpha}}{\Gamma(\alpha+1)}+4 \frac{t^{2 \alpha}}{\Gamma(2 \alpha+1)} \\
& +8 \frac{t^{3 \alpha}}{\Gamma(3 \alpha+1)}+16 \frac{t^{4 \alpha}}{\Gamma(4 \alpha+1)}+\cdots
\end{aligned}
$$

Then, we find the following data:

$$
\begin{gathered}
f^{\prime}(0)=2, \quad f^{\prime \prime \prime}(0)=-2, \quad f^{(v)}(0)=2, \\
f^{(v i i)}(0)=-2, \ldots .
\end{gathered}
$$

Next, using (50) and (54), we have the Taylor series expansion of $f(x)$ as follows:

$$
\begin{aligned}
f(x)= & f(0)+f^{\prime}(0) x+f^{\prime \prime}(0) \frac{x^{2}}{2 !} \\
& +f^{\prime \prime \prime}(0) \frac{x^{3}}{3 !}+f^{(i v)}(0) \frac{x^{4}}{4 !}+\cdots \\
= & 2 x-2 \frac{x^{3}}{3 !}+2 \frac{x^{5}}{5 !}+2 \frac{x^{7}}{7 !}+\cdots .
\end{aligned}
$$

That is,

$$
f(x)=2\left[x-\frac{x^{3}}{3 !}+\frac{x^{5}}{5 !}+\frac{x^{7}}{7 !}+\cdots\right]
$$

which is the series expansion of the function $2 \sin x$. Consequently, we determine the source function $f(x)$ as

$$
f(x)=2 \sin x
$$

Example 2. We consider the inverse problem of determining source function $f(t)$ in the following one-dimensional fractional heat-like diffusion equation:

$$
\begin{array}{r}
D_{t}^{\alpha} u(x, t)=\frac{1}{2} x^{2} u_{x x}(x, t)+f(t), \\
x>0, \quad 0<\alpha \leq 1, \quad t>0,
\end{array}
$$

subject to following initial and mixed boundary conditions

$$
u(x, 0)=x^{2}+\frac{1}{2}, \quad u(0, t)=\frac{e^{2 t}}{2}, \quad u_{x}(0, t)=0 .
$$

Now let us determine the source function $f(t)$. To reduce the problem, we define new functions as follows:

$$
\begin{gathered}
w(t)=J_{t}^{\alpha} f(t), \\
u(x, t)=v(x, t)+w(t) .
\end{gathered}
$$

Then, our reduced problem is given as follows:

$$
\begin{gathered}
D_{t}^{\alpha} v(x, t)=\frac{1}{2} x^{2} v_{x x}(x, t), \quad 0<\alpha \leq 1, t>0 \\
v(x, 0)=u(x, 0)-w(0)=x^{2}+\frac{1}{2}, \\
v(0, t)=u(0, t)-w(t)=\frac{e^{2 t}}{2}-w(t), \\
v_{x}(0, t)=0 .
\end{gathered}
$$

Applying $J_{t}^{\alpha}$ to both sides of (61), then we get

$$
v(x, t)-v(x, 0)=\frac{1}{2} x^{2} J_{t}^{\alpha} v_{x x}(x, t)
$$

which implies

$$
v(x, t)=x^{2}+\frac{1}{2}+\frac{1}{2} x^{2} J_{t}^{\alpha} v_{x x}(x, t) .
$$

By using ADM for (63), we obtain

$$
\begin{gathered}
v_{0}=x^{2}+\frac{1}{2} \\
v_{k+1}=\frac{1}{2} x^{2} J_{t}^{\alpha}\left(v_{k}\right)_{x x}(x, t), \quad k \geq 0 .
\end{gathered}
$$

Then, for $k=0$, we get

$$
\begin{aligned}
v_{1} & =\frac{1}{2} x^{2} J_{t}^{\alpha}\left(v_{0}\right)_{x x}(x, t) \\
& =x^{2} \frac{t^{\alpha}}{\Gamma(\alpha+1)},
\end{aligned}
$$

similarly, for $k=1$, we get

$$
\begin{aligned}
v_{2} & =\frac{1}{2} x^{2} J_{t}^{\alpha}\left(v_{1}\right)_{x x}(x, t) \\
& =x^{2} \frac{t^{2 \alpha}}{\Gamma(2 \alpha+1)},
\end{aligned}
$$


and for $k=2$, we get

$$
\begin{aligned}
v_{3} & =\frac{1}{2} x^{2} J_{t}^{\alpha}\left(v_{2}\right)_{x x}(x, t) \\
& =x^{2} \frac{t^{3 \alpha}}{\Gamma(3 \alpha+1)},
\end{aligned}
$$

As a result, we get the solution $v$ as follows:

$$
\begin{aligned}
v(x, t)= & v_{0}+v_{1}+v_{2}+v_{3}+\cdots \\
= & x^{2}+\frac{1}{2}+x^{2} \frac{t^{\alpha}}{\Gamma(\alpha+1)} \\
& +x^{2} \frac{t^{2 \alpha}}{\Gamma(2 \alpha+1)}+x^{2} \frac{t^{3 \alpha}}{\Gamma(3 \alpha+1)}+\cdots \\
= & \frac{1}{2}+x^{2}\left[1+\frac{t^{\alpha}}{\Gamma(\alpha+1)}+\frac{t^{2 \alpha}}{\Gamma(2 \alpha+1)}\right. \\
& \left.+\frac{t^{3 \alpha}}{\Gamma(3 \alpha+1)}+\cdots\right] .
\end{aligned}
$$

Therefore, from the boundary condition we have

$$
w(t)=\frac{e^{2 t}}{2}-\frac{1}{2}
$$

Using (69) in the definition $D_{t}^{\alpha} w(t)=D_{t}^{\alpha} J_{t}^{\alpha} f(t)=$ $f(t)$, finally we obtain the source function $f(t)$ as $f(t)=$ $D_{t}^{\alpha}\left(\left(e^{2 t} / 2\right)-(1 / 2)\right)$; that is,

$$
f(t)=\frac{1}{2} D_{t}^{\alpha}\left(e^{2 t}\right) .
$$

Here,

$$
D_{t}^{\alpha}\left(e^{2 t}\right)=t^{-\alpha} E_{1,1-\alpha}(2 t),
$$

where $E_{1,1-\alpha}$ is Mittag-Leffler function with two parameters given as; (6).

\section{Conclusion}

The best part of this method is that one can easily apply ADM to the fractional partial differential equations like applying ADM to ordinary differential equations.

\section{References}

[1] A. A. Kilbas, H. M. Srivastava, and J. J. Trujillo, Theory and Applications of Fractional Differential Equations, vol. 204 of North-Holland Mathematics Studies, Elsevier Science B.V., Amsterdam, The Netherlands, 2006.

[2] K. B. Oldham and J. Spanier, The Fractional Calculus, Academic Press, New York, NY, USA, 1974.

[3] K. S. Miller and B. Ross, An Introduction to the Fractional Calculus and Fractional Differential Equations, A Wiley-Interscience Publication, John Wiley \& Sons, New York, NY, USA, 1993.
[4] I. Podlubny, Fractional Differential Equations, vol. 198 of Mathematics in Science and Engineering, Academic Press, San Diego, Calif, USA, 1999.

[5] S. S. Ray, K. S. Chaudhuri, and R. K. Bera, "Analytical approximate solution of nonlinear dynamic system containing fractional derivative by modified decomposition method," Applied Mathematics and Computation, vol. 182, no. 1, pp. 544-552, 2006.

[6] K. Diethelm, "An algorithm for the numerical solution of differential equations of fractional order," Electronic Transactions on Numerical Analysis, vol. 5, no. Mar., pp. 1-6, 1997.

[7] D. Baleanu, K. Diethelm, E. Scalas, and J. J. Trujillo, Fractional Calculus Models and Numerical Methods, vol. 3 of Series on Complexity, Nonlinearity and Chaos, World Scientific Publishing, Hackensack, NJ, USA, 2012.

[8] M. Alipour and D. Baleanu, "Approximate analytical solution for nonlinear system of fractional differential equations by BPs operational matrices," Advances in Mathematical Physics, vol. 2013, Article ID 954015, 9 pages, 2013.

[9] G. Adomian, Solving Frontier Problems of Physics: The Decomposition Method, vol. 60 of Fundamental Theories of Physics, Kluwer Academic Publishers, Dordrecht, The Netherlands, 1994.

[10] G. Adomian, "Solutions of nonlinear P. D. E," Applied Mathematics Letters, vol. 11, no. 3, pp. 121-123, 1998.

[11] K. Abbaoui and Y. Cherruault, "The decomposition method applied to the Cauchy problem," Kybernetes, vol. 28, no. 1, pp. 68-74, 1999.

[12] D. Kaya and A. Yokus, "A numerical comparison of partial solutions in the decomposition method for linear and nonlinear partial differential equations," Mathematics and Computers in Simulation, vol. 60, no. 6, pp. 507-512, 2002.

[13] D. A. Murio, "Time fractional IHCP with Caputo fractional derivatives," Computers \& Mathematics with Applications, vol. 56, no. 9, pp. 2371-2381, 2008.

[14] A. N. Bondarenko and D. S. Ivaschenko, "Numerical methods for solving inverse problems for time fractional diffusion equation with variable coefficient," Journal of Inverse and Ill-Posed Problems, vol. 17, no. 5, pp. 419-440, 2009.

[15] Y. Zhang and X. Xu, "Inverse source problem for a fractional diffusion equation," Inverse Problems, vol. 27, no. 3, Article ID 035010, 12 pages, 2011.

[16] M. Kirane and S. A. Malik, "Determination of an unknown source term and the temperature distribution for the linear heat equation involving fractional derivative in time," Applied Mathematics and Computation, vol. 218, no. 1, pp. 163-170, 2011.

[17] M. Caputo, "Linear models of dissipation whose Q is almost frequency independent-II," Geophysical Journal International, vol. 13, no. 5, pp. 529-539, 1967.

[18] S. T. Mohyud-Din, A. Ylldırım, and M. Usman, "Homotopy analysis method for fractional partial differential equations," International Journal of Physical Sciences, vol. 6, no. 1, pp. 136145, 2011.

[19] G. Mittag-Leffler, "Sur la représentation analytique d'une branche uniforme d'une fonction monogène," Acta Mathematica, vol. 29, no. 1, pp. 101-181, 1905. 


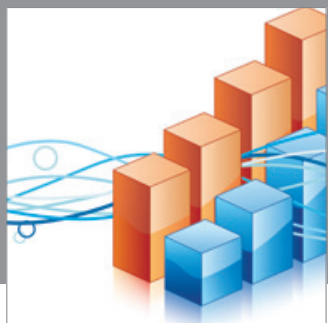

Advances in

Operations Research

mansans

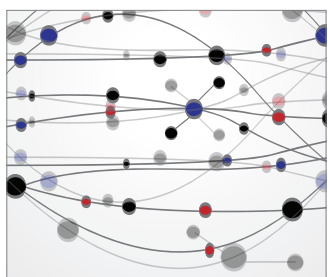

The Scientific World Journal
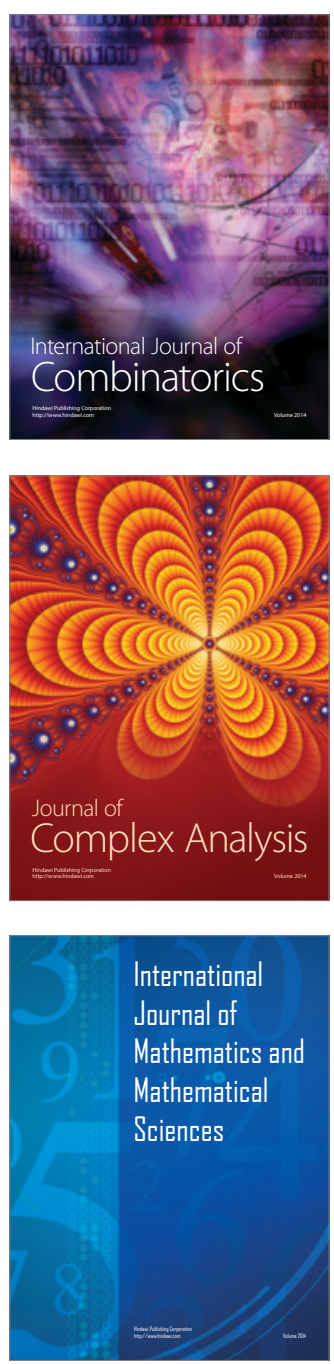
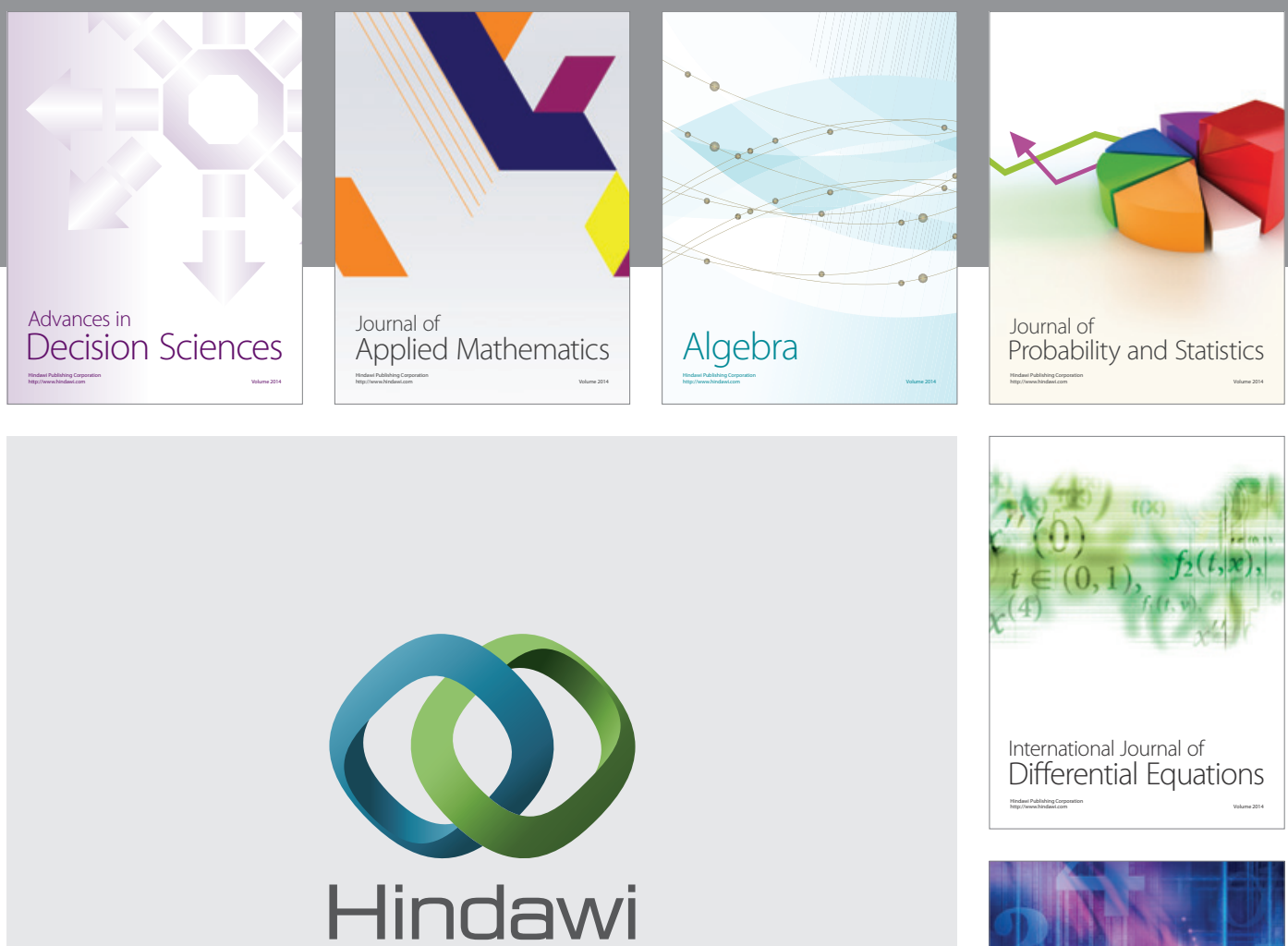

Submit your manuscripts at http://www.hindawi.com
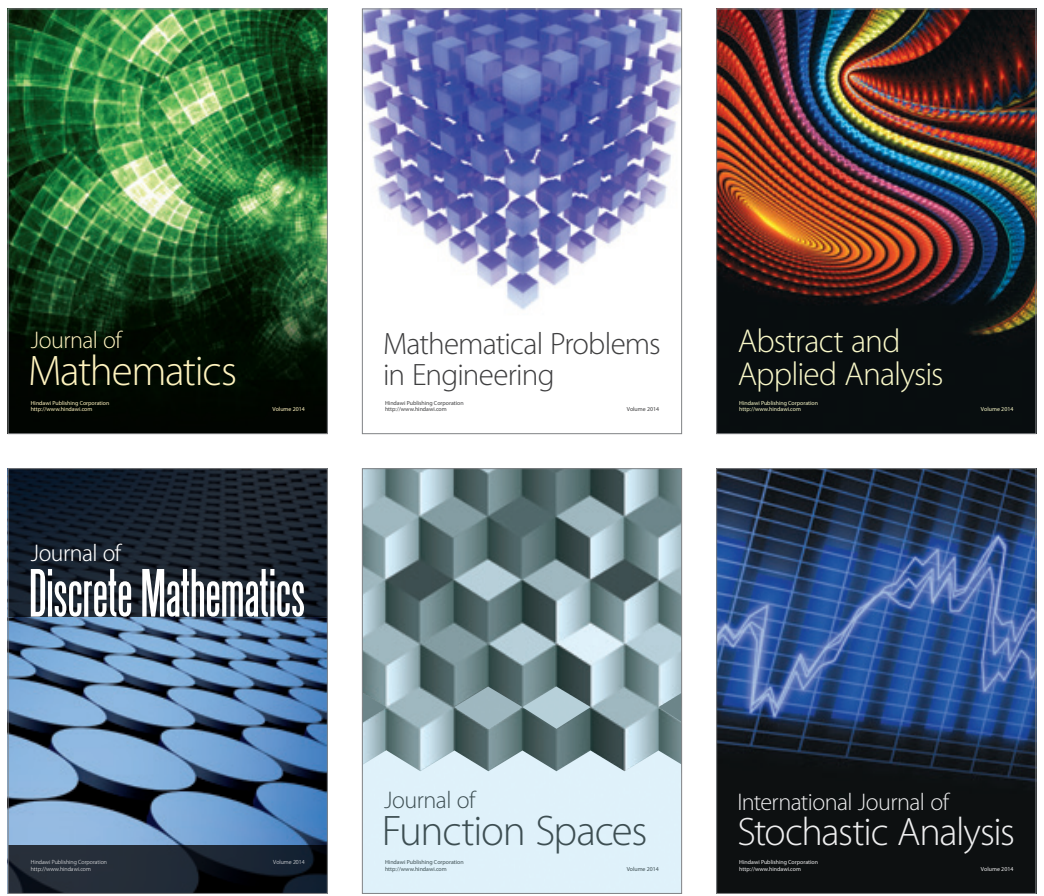

Journal of

Function Spaces

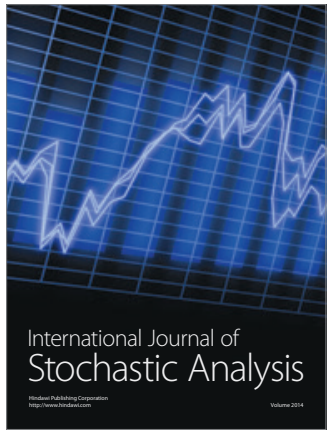

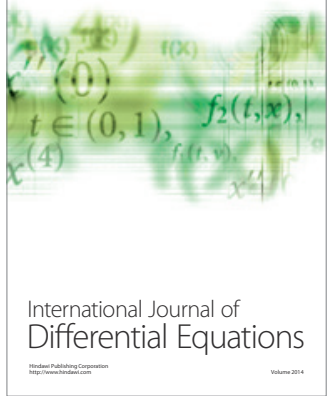
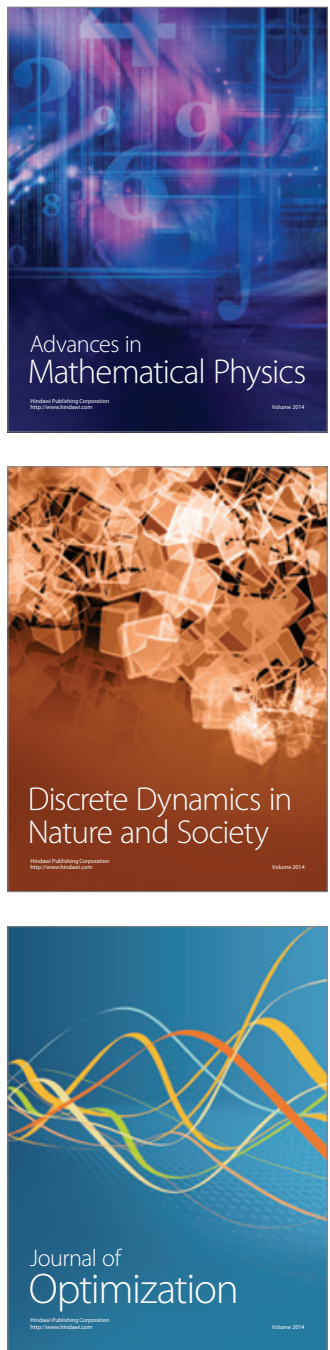